\title{
Extending Transmission Distance of High-Density WDM Systems Using Post Transmitter Fiber Raman Amplifiers
}

\author{
Ming-Seng Kao and Jingshown Wu
}

\begin{abstract}
We investigate the behavior of the multichannel signals in a high-density WDM system with the consideration of stimulated Raman scattering. We provide a parameter to evaluate the depth of $\mathrm{Ra}$ man crosstalk and analyze the transmission distance limitation imposed by Raman crosstalk. To extend the system transmission distance, a post transmitter fiber Raman amplifier is employed. The allocation of signal channels on the pump gain profile is discussed. We found that there exists an optimum pump power which results in a maximum amplifier gain. The example shows that the transmission distance limitation imposed by Raman crosstalk can be extended by $20 \mathrm{~km}$ for a 30channel system with $0.25-\mathrm{dB} / \mathbf{k m}$ fiber loss.
\end{abstract}

\section{INTRODUCTION}

QINGLE-MODE optical fibers present various nonlinear opDical effects due to their small cross section. These optical nonlinearities can be used for many device functions and useful optical components [1]. However, they also present limitation on optical fiber systems, in particular on multichannel systems, because of nonlinear conversion of lightwaves [2], [3]. At present the high-density wavelength division multiplexing (HDWDM) technique is a potential candidate to fully exploit the vast bandwidth provided by a single-mode fiber [4], the limitation caused by optical nonlinearities in an HDWDM system is an important issue [5]. Stimulated Raman scattering (SRS) with its much wider bandwidth than stimulated Brillouin scattering is likely to be a critical limitation on an HDWDM system [6], [7]. On the other hand, direct optical amplification by using SRS [8], [9], called fiber Raman amplifier, which eliminates the need of optical/electrical/optical conversion, wavelength multiplexing, and complicated electronics in conventional optical repeaters, is of great interest in a multichannel system [10][12].

This paper investigates the application of post transmitter fiber Raman amplifier (PTFRA) in an HDWDM system. The PTFRA is formed by simply employing a strong pump at the transmitting end as a Raman amplifier to boost the signals in an HDWDM system. The idea stems from two viewpoints. First, with a PTFRA the signal power is expected to increase by obtaining power from the pump via SRS so that the system transmission distance can be extended. Second, since tens of signal channels are transmitted, the crosstalk caused by SRS is known to be a limiting factor for an HDWDM system. In an intensity

Manuscript received May 22, 1990. This work was supported in part by the National Science Council and Telecommunication Laboratories of ROC under contract TL-NSC-79-013.

M.-S. Kao is with the Department of Communication Engineering, National Chiao Tung University, Hsinchu, Taiwan, Republic of China.

$J$. Wu is with the Department of Electrical Engineering, National Taiwan University, Taipei, Taiwan, Republic of China.

IEEE Log Number 9041779. modulated HDWDM system, which is considered here, the Raman crosstalk is manifested as the power depletion of those shorter wavelength channels and most severe for the shortest wavelength channel [6]. So that the signal power of the shortest wavelength channel is carried away which limits the system transmission distance. By employing a PTFRA, the depleted powers, in particular that of the shortest wavelength channel, can be compensated. Therefore the Raman crosstalk limitation can be extended.

In Section II, we study Raman crosstalk in an HDWDM system. The dependence of Raman crosstalk on the system parameters including signal powers, channel numbers, and channel spacings is illustrated. In Section III we investigate the signal propagation with and without a PTFRA. We have discussed the influence of channel allocation within the pump gain profile in Section IV. An optimum pump power which results in a maximum amplifier gain is shown. We also estimate the extension of transmission distance with the use of PTFRA and finally conclude the article.

\section{Raman Crosstalk}

Here we consider the crosstalk caused by SRS in an N-channel HDWDM fiber transmission system. Let $A$ and $\alpha$ denote the effective core area and loss coefficient of the fiber, respectively. The effective fiber core diameter can be calculated through appropriate overlap integrals. Here we assume a $6-\mu \mathrm{m}$ effective core diameter and take $\alpha$ as $0.25 \mathrm{~dB} / \mathrm{km}$. We use a piecewise linear function as shown in Fig. 1 to approximate the measured gain profile [13] and take the Raman gain peak as $8 \times 10^{-14}$ $\mathrm{m} /$ W at Raman shift $v_{R}=440 \mathrm{~cm}^{-1}\left(1 \mathrm{~cm}^{-1} \equiv 30 \mathrm{GHz}\right)$.

In an HDWDM system the signal linewidths are much smaller than the bandwidth of Raman gain profile (about $58 \mathrm{~nm}$ at 1.55 $\mu \mathrm{m}$ ), hence the Raman gain for a signal channel can be taken as a constant. The Raman gain constant coupling the $i$ th and $m$ th signal channels $g_{i m}$ can then be obtained from the piecewise linear function shown in Fig. 1 with Raman shift $v_{R}=v_{s i}-$ $v_{s m}$, where $v_{s i}$ and $v_{s m}$ denote the lightwave frequencies of the $i$ th and $m$ th channels and $v_{s i}>v_{s m}$.

Let the signal powers $S_{i}(0)(i=1,2, \cdots N)$ be injected at $z=0$ and travel in the $+z$ direction. We assume all the signals are pulse modulated and deal with the worst case that all the channels are in the ON state. In a pulse modulated HDWDM system, fiber dispersion can cause group velocity mismatch among the different wavelength signal pulses which results in walk-off effect in the Raman processes. However, a system with pulse duration beyond about a nanosecond, where the effect of group velocity mismatch is essentially negligible, can be treated as a continuous wave (CW) case [14]. For high-speed systems 


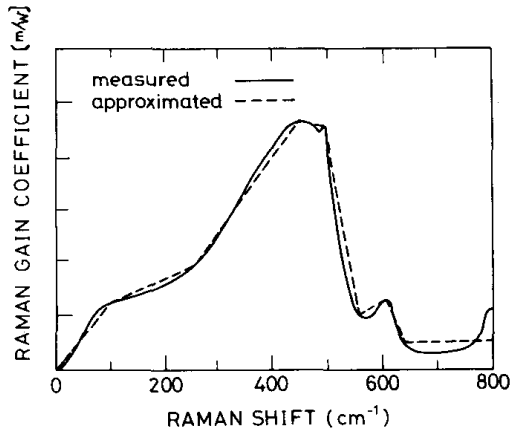

Fig. 1. The measured and approximated Raman gain profile in fibers.

operating in subnanosecond range, the group velocity mismatch among the signals can somewhat reduce the mutual Raman interactions so that the crosstalk decreases. Here for simplicity we merely consider a system with pulse duration beyond a nanosecond so that the Raman interactions are treated as a CW case. For the high-speed systems with pulse duration less than a nanosecond the results of this paper can be viewed as an overestimate.

Considering Raman crosstalk, the coupled equations governing the propagation of the multiwavelength signals are formulated as

$$
\begin{gathered}
\frac{d S_{i}(z)}{d z}=\left[-\alpha+\sum_{j=1}^{i-1} \frac{g_{j i} S_{j}(z)}{2 A}-\sum_{k=i+1}^{N} \frac{v_{s i}}{v_{s k}} \frac{g_{i k} S_{k}(z)}{2 A}\right] S_{i}(z) \\
i=1,2, \cdots N
\end{gathered}
$$

where we assume $v_{s 1}>v_{s 2}, \cdots>v_{s N}$ and the $N$ channels are equally spaced with channel spacing $\Delta v$. The second and the third terms in the parenthesis of (1) should be omitted for $i=$ 1 and $i=N$, respectively. The first term denotes the fiber loss while the second and the third terms express the cross coupling among the signal channels. The factor 2 in the second and the third terms of the denominator accounts for the random polarization of the signal waves [15].

These cross coupling terms results in crosstalk among the signals. As a result, optical powers of the shorter wavelength channels will be depleted by the longer wavelength channels. This occurs most severe for the shortest wavelength channel, Channel 1 ( $\mathrm{CH} \mathrm{1).} \mathrm{Therefore,} \mathrm{the} \mathrm{transmission} \mathrm{distance} \mathrm{will} \mathrm{be} \mathrm{lim-}$ ited by $\mathrm{CH} 1$ if equal signal powers are launched at the transmitting end. For the sake of clarity, we consider equal transmitted signal powers hereafter. We introduce a variable change to simplify the coupling equations. Let

$$
S_{i}(z)=\exp \left(V_{i}(z)-\alpha z\right)
$$

where the term $e^{-\alpha z}$ accounts for linear fiber loss and $e^{V_{i(z)}} \mathrm{de}$ scribes the variation of the $i$ th signal power due to mutual couplings. If no Raman interaction exists, $e^{V_{i}(z)}$ is a constant. We have

$$
\frac{d S_{i}(z)}{d z}=\left(\frac{d V_{i}(z)}{d z}-\alpha\right) S_{i}(z)
$$

substituting (3) into (1) becomes

$$
\frac{d V_{i}(z)}{d z}=\left[\sum_{j=1}^{i-1} \frac{g_{j i} e^{V_{j}(z)}}{2 A}-\sum_{k=i+1}^{N} \frac{v_{s i}}{v_{s k}} \frac{g_{i k} e^{V_{k}(z)}}{2 A}\right] e^{-\alpha z} .
$$

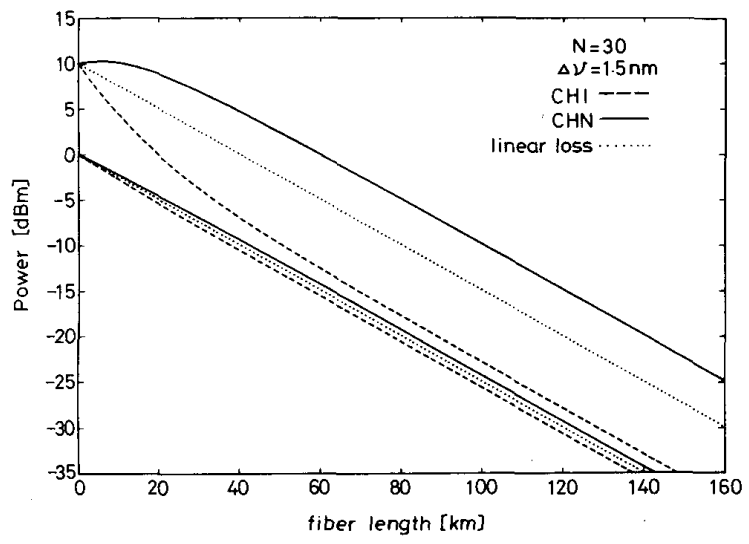

Fig. 2. The variation of signal powers versus fiber length. For familiarity we use wavelength unit for $\Delta v$.

In the region far from the transmitting end $e^{-\alpha z} \simeq 0$ then

$$
\frac{d V_{i}(z)}{d z} \simeq 0
$$

From (3) and (5) it is apparent that in the far end region all the signals are expected to propagate nearly with fiber loss and experience little cross coupling because of low signal levels. Fig. 2 shows the variation of signal powers along a fiber for $\mathrm{CH} 1$ and $\mathrm{CH} \mathrm{N}$. In addition to fiber loss, $\mathrm{CH} 1$, being the shortest wavelength channel, is depleted by the other channels due to Raman crosstalk while $\mathrm{CH} \mathrm{N}$, being the longest wavelength channel, obtains power from the other channels particularly when the initial signal powers are large. It is clear that $\mathrm{CH} 1$ will limit system transmission distance. Hence, in the following we consider CH 1 only. At the transmitting end $(z=0), e^{-\alpha z}$ $=1$, then

$$
\left.\frac{d V_{i}(z)}{d z}\right|_{z=0}=\left[\sum_{j=1}^{i-1} \frac{g_{j i} e^{V_{j}(0)}}{2 A}-\sum_{k=i+1}^{N} \frac{v_{s i}}{v_{s k}} \frac{g_{i k} e^{V_{k}(0)}}{2 A}\right] .
$$

Let $S_{0}$ denote the initial transmitted signal power for the channels, i.e., $S_{i}(0)=S_{0}$. Since $S_{i}(0)=e^{V_{i}(0)}$, expression $(6)$ for $\mathrm{CH} 1$ is written as

$$
\left.\frac{d V_{1}(z)}{d z}\right|_{z=0}=-S_{0} C_{1}
$$

where

$$
C_{\mathbf{l}}=\sum_{k=2}^{N} \frac{v_{s 1}}{v_{s k}} \frac{g_{1 k}}{2 A}
$$

Expression (8) indicates that $C_{1}$ is a system parameter which depends on the number of channels, signal frequencies, cross coupling constants, as well as the effective core area. If $C_{1} S_{0}$ $\ll \alpha$, it is apparent from (3) that $S_{1}(z)$ will propagate nearly with fiber loss and negligible cross coupling. In this case the Raman crosstalk is small. For the case where $C_{1} S_{0} \gg \alpha$, it is expected that strong cross coupling occurs so that $\mathrm{CH} 1$ is severely depleted by the other channels. We define a parameter $R$ as

$$
R=\frac{C_{1} S_{0}}{\alpha}
$$




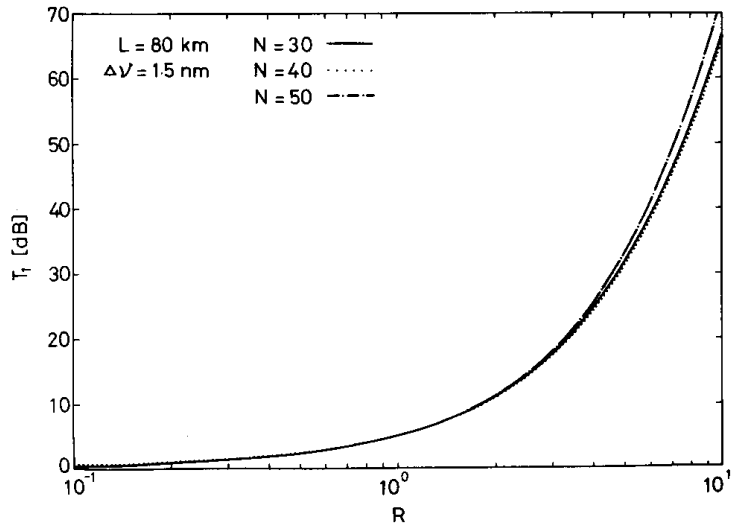

Fig. 3. The relation between $T_{1}$ and $R$ for several channel numbers.

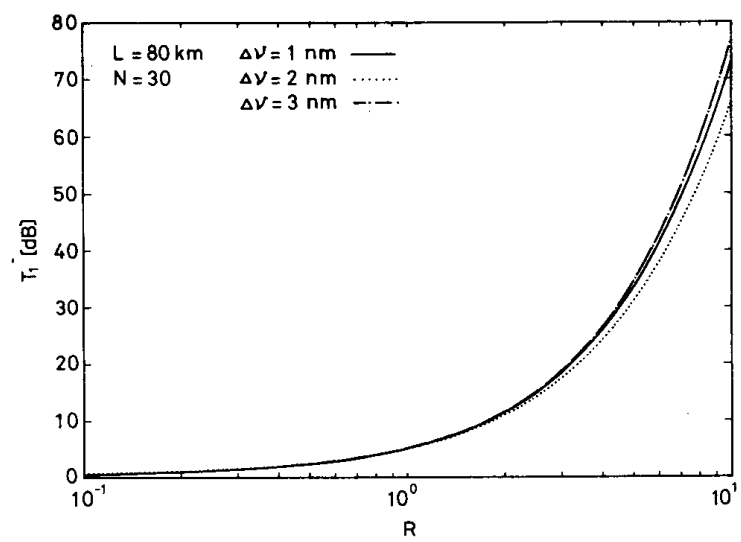

Fig. 4. The relation between $T_{1}$ and $R$ for several channel spacings.

which is essentially the ratio of cross coupling to fiber loss for $\mathrm{CH}$ 1. We also define a crosstalk coefficient for $\mathrm{CH} 1$ as

$$
T_{1}=-10 \log \frac{S_{1}(L)}{S_{0} e^{-\alpha L}}
$$

where $S_{1}(L)$ is the received signal power of $\mathrm{CH} 1$ at $z=L$.

Figs. 3 and 4 show the relation between $T_{1}$ and $R$ for various channel numbers and channel spacings, respectively. Here numerical analyses are carried out to solve the coupling equations. In the examples $S_{0}$ is equal to $R \alpha / C_{1}$ where $C_{1}$ is calculated from (8). We see that $T_{1}$ increases with $R$ and increases rapidly when $R>1$. Hence, the parameter $R$ can be used to evaluate the depth of Raman crosstalk. For a system to operate with little Raman crosstalk, the parameter should be kept small.

There exists a maximum received signal power for $\mathrm{CH} 1$, as depicted in Fig. 5, at a distance $L$ from the transmitting end. At low transmitted signal power level, $S_{1}(L)$ increases with $S_{0}$; after reaching a maximum value, $S_{1}(L)$ decreases as $S_{0}$ further increases. This is due to Raman crosstalk and we cannot further increase the received signal power if the maximum point is reached. Therefore the system transmission distance is limited. The departure from the linear loss curve accounts for the amount of Raman crosstalk. Depending on system parameters, the maxima occur at different transmitted signal levels as shown in Fig. 6. This limitation can be relaxed if narrow channel spacing is

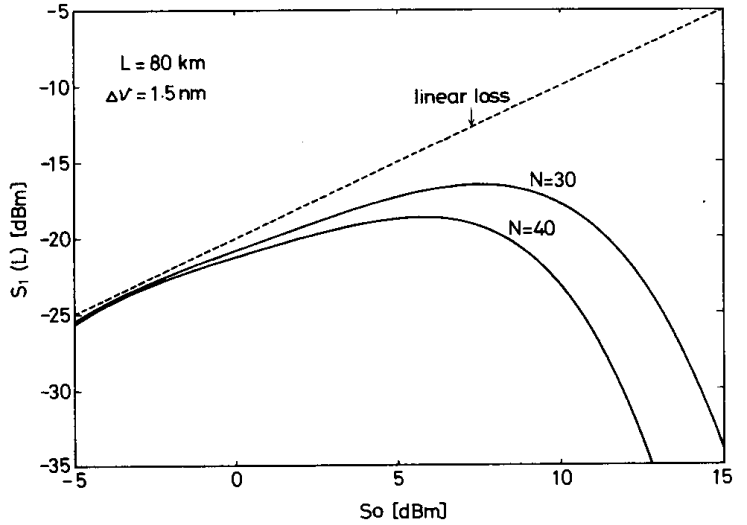

Fig. 5. The received versus transmitted signal power of $\mathrm{CH} 1$ for two channel numbers.

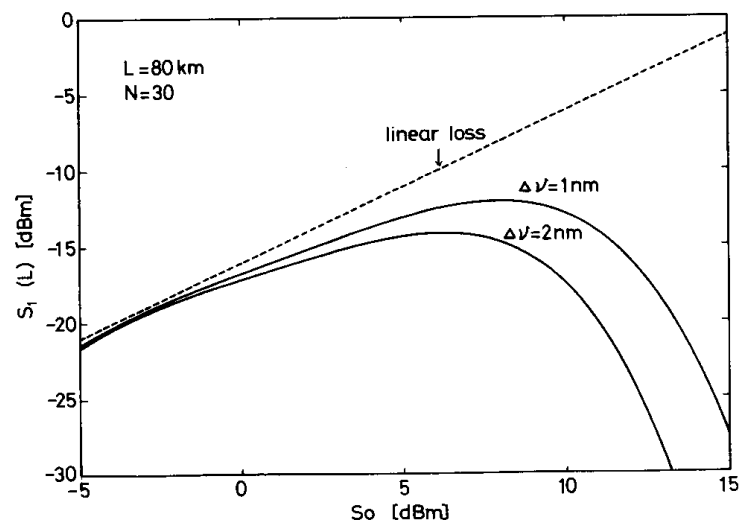

Fig. 6. The received versus transmitted signal powers of $\mathrm{CH} 1$ for two channel spacings.

used. But it requires narrower linewidth DFB lasers and higher resolution wavelength demultiplexers which restrict the use of such scheme.

\section{Signal Propagation in An HDWDM System WITH PTFRA}

In this section we consider the use of PTFRA to extend the transmission distance limitation imposed by Raman crosstalk. The PTFRA can be constructed by employing a strong continuous pump light at the transmitting end. The pump frequency $v_{p}$ is chosen so that $v_{p}>v_{s 1}$, hence optical power of the pump can be coupled to the signals through SRS. With a strong pump power $P(0)$ injected at $z=0$ with the signals, the coupled equations become

$$
\begin{aligned}
\frac{d S_{i}(z)}{d z}= & {\left[-\alpha+\frac{g_{i}}{2 A} P(z)+\sum_{j=1}^{i-1} \frac{g_{j i} S_{j}(z)}{2 A}\right.} \\
& \left.-\sum_{k=i+1}^{N} \frac{v_{s i}}{v_{s k}} \frac{g_{i k} S_{k}(z)}{2 A}\right] S_{i}(z), \quad i=1,2, \cdots N
\end{aligned}
$$

$\frac{d P(z)}{d z}=-\left[\alpha+\sum_{i=1}^{N} \frac{v_{p}}{v_{s i}} \frac{g_{i} S_{i}(z)}{2 A}\right] P(z)$ 


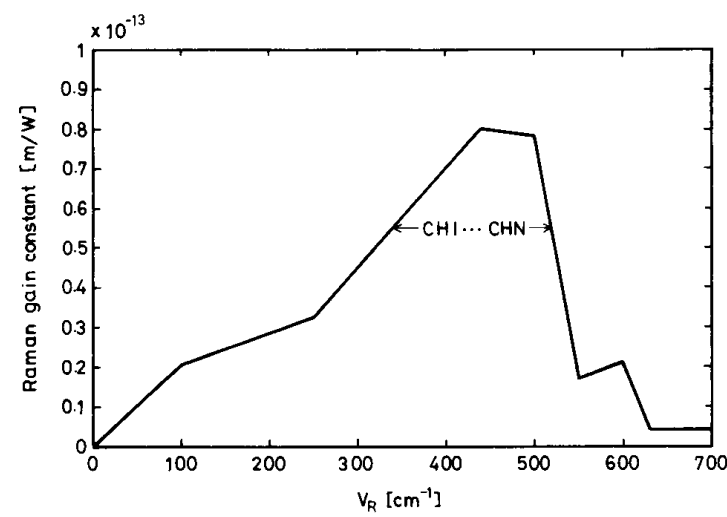

(a)

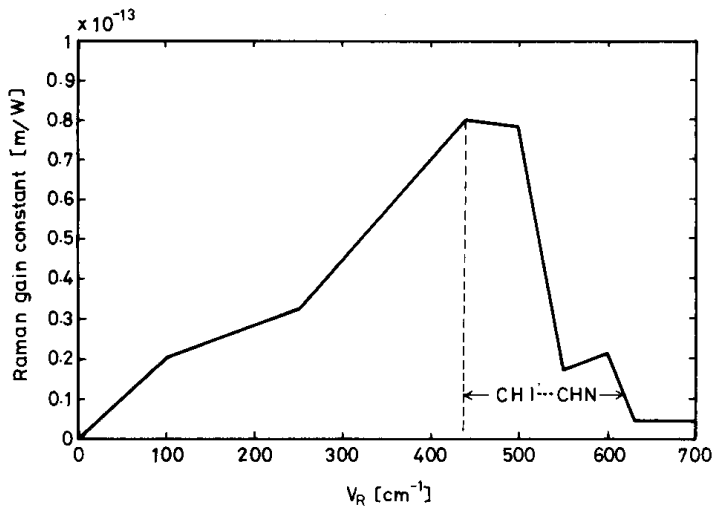

(b)

Fig. 7. The allocation of signal channels within the pump gain profile. (a) Place the channels within the high-gain region. (b) Locate $\mathrm{CH} 1$ at the gain peak.

where $g_{i}$ is the Raman gain constant between the pump and the $i$ th signal which can be obtained from Fig. 1 with $v_{R}=v_{p}$ $v_{s i}$.

Every signal channel is expected to gain power from the pump through the coupling constant $g_{i}^{\prime} \mathrm{s}$ so that the allocation of the signals within the pump Raman gain profile, which determines $g_{i}^{\prime} \mathrm{s}$, will therefore strongly influence the PTFRA performance. A straightforward assignment is shown in Fig. 7(a) in which the signals are placed at the high-gain region of pump gain profile. With such assignment, the pump power is expected to be fully utilized. In this case $g_{1}=g_{N}$ and they are the smallest gain constant coupling with the pump. Thus $\mathrm{CH} 1$ is still the lowest power channel since it gains least power from the pump and is depleted most by the other channels.

The relation between the transmitted and received signal powers at $z=L$ for $\mathrm{CH} 1$ with and without a PTFRA is shown in Fig. 8. In the example we see that the received signal power with a PTFRA, in comparison with that without a PTFRA $(P(0)=0 \mathrm{~W})$, increases only for $P(0)=0.5 \mathrm{~W}$ and $S_{0}<$ $-0.5 \mathrm{dBm}$, and decreases elsewhere. It is interesting to see that the use of larger pump power results in less the signal power of $\mathrm{CH} 1$ at $z=L$. This can be explained by Fig. 9 where the distribution of $\mathrm{CH} 1$ along the fiber is depicted. For $P(0)=$ $0.7 \mathrm{~W}$, in the region near $z=0, \mathrm{CH} 1$ gains more power from the pump compared with the case $P(0)=0.5 \mathrm{~W}$. Since the

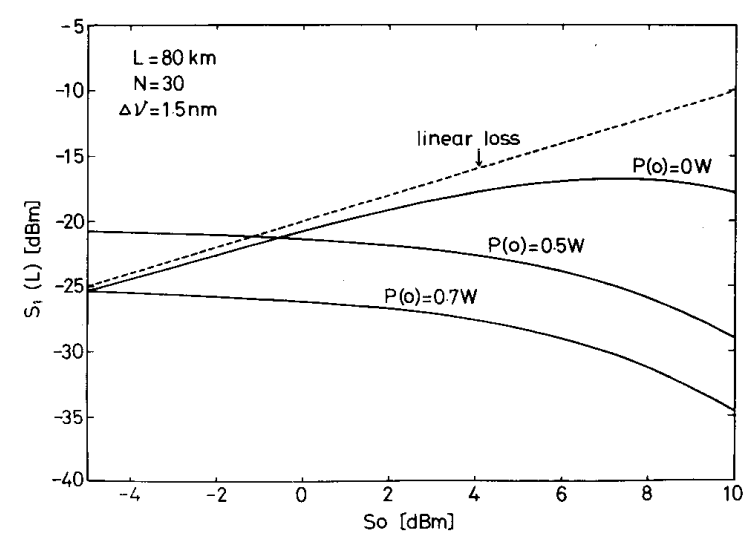

Fig. 8. The received and transmitted signal power of $\mathrm{CH} 1$ with and with out a PTFRA.

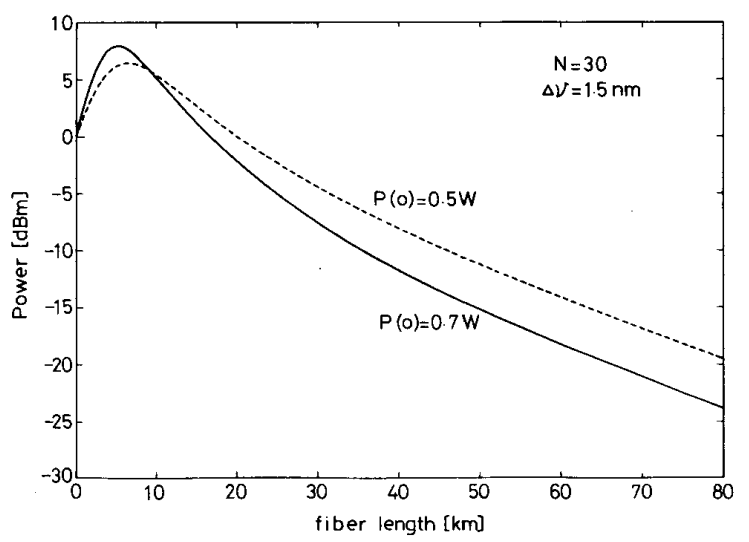

Fig. 9. The power variation of $\mathbf{C H} 1$ along the fiber for two pump powers.

other channels are also amplified to a high-power level which give rise to strong Raman interaction among the signal channels, so that after reaching its maximum point $\mathrm{CH} 1$ is severely depleted by the other channels. As a result, the received power at $z=L$ for $P(0)=0.7 \mathrm{~W}$ is less than that for $P(0)=0.5$ W.

According to the above results we conclude that the use of PTFRA can increase little, or even decrease, the received signal power of $\mathrm{CH} 1$ if the signals are allocated as shown in Fig. 7(a). With such arrangement the pump power can be fully utilized but $\mathrm{CH} 1$, being the mostly depleted channel, gains least power from the pump and experiences stronger depletion by the other channels so that the result is not desirable. Thus we look for an alternative, as shown in Fig. 7(b), by allocating $\mathrm{CH} 1$ at the Raman gain peak. In this case $\mathrm{CH} 1$ gains much power from the pump because $g_{1}$ is the largest while $\mathrm{CH} \mathrm{N}$ gains little power from the pump because $g_{N}$ is the smallest. Because the highpower pump has strong couple with $\mathrm{CH} 1$ so that $\mathrm{CH} 1$ may no longer be the limiting channel of the system, we consider two extreme cases to determine the minimum signal power of the system. One is the same case as before that all the channels are ON. In this case the least signal power at $z=L$ will be obtained by numerically solving the coupling equations. The other is the case where only one channel is on and the other channels are OFF. In this case only the channel in the oN state is amplified 


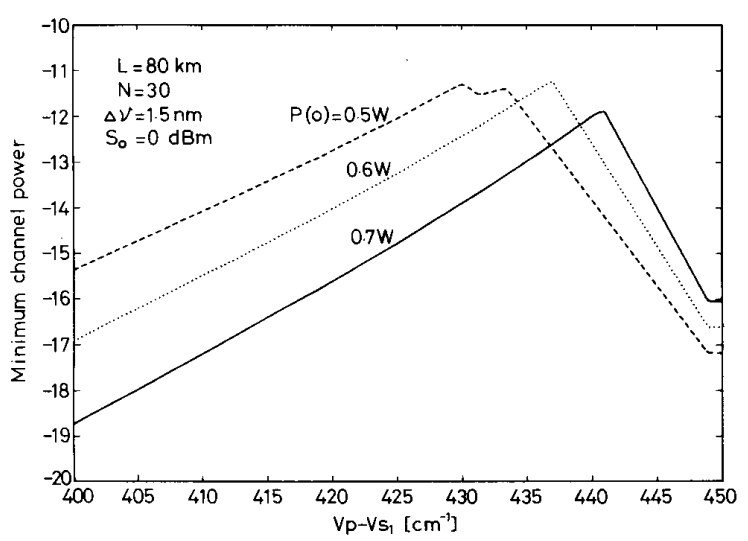

Fig. 10. The relation between the minimum received signal power and $v_{p}$ $-v_{s 1}$ for several pump powers. Note the gain peak at $440 \mathrm{~cm}^{-1}$.

by the pump and because $g_{N}$ is the smallest so that $\mathrm{CH} \mathrm{N}$ will be the limiting channel of the system. And the minimum signal power is determined by the lesser one of these two extreme conditions. When only $\mathrm{CH} \mathrm{N}$ is oN, the couple equations reduces to single channel case which can be analytically presented as [16]

$$
S_{N}(z)=\frac{S_{0} \exp \left[K\left(1-e^{-\alpha z}\right)-\alpha z\right]}{1+\frac{v_{p} S_{0}}{v_{s N} P(0)} \exp \left[K\left(1-e^{-\alpha z}\right)\right]}
$$

where

$$
K=\frac{g_{N} P(0)}{\alpha A} .
$$

The idea of allocating $\mathrm{CH} 1$ at the pump gain peak is with the view to compensate its depleted power from the pump, which may not be the optimum choice since $\mathrm{CH} \mathrm{N}$ obtains little power from the pump which may be the limiting channel of the system when the other channels are OFF. To investigate the validity of this idea we have tried other arrangements in Fig. 10 by offsetting $v_{p}-v_{s 1}$ from the gain peak $v_{R}=440 \mathrm{~cm}^{-1}$. Due to the piecewise linear approximation of the Raman gain profile, the results are not smooth. It is found that although the placement of CH 1 at the gain peak is not the optimum choice for the various pump powers but it indeed has little departure from the optimum value. We further plot the same relation in Fig. 11 for two transmitted signal powers. Again the optimum placement of $\mathrm{CH} 1$ deviates little from the gain peak. Thus the placement of $\mathrm{CH} 1$ at the Raman gain peak of the pump can be viewed as a suboptimum choice and the optimum choice should be numerically calculated around the vicinity of the gain peak.

We define the amplifier gain at $z=L$ as

$$
G=10 \log \frac{S^{\prime}(L)}{S_{1}(L)}
$$

where $S_{1}(L)$ denotes the received power of $\mathrm{CH} 1$ at $z=L$ without a PTFRA and $S^{\prime}(L)$ is the minimum signal power when a PTFRA is employed. An example is illustrated in Fig. 12 where three transmitted signal levels are provided. It is clear that over $10 \mathrm{~dB}$ gain can be achieved for $S_{0}=-5 \mathrm{dBm}$ but little gain obtainable when $S_{0}=5 \mathrm{dBm}$. Also note that for a given $S_{0}$ there exists an optimum pump power such that a maximum gain can

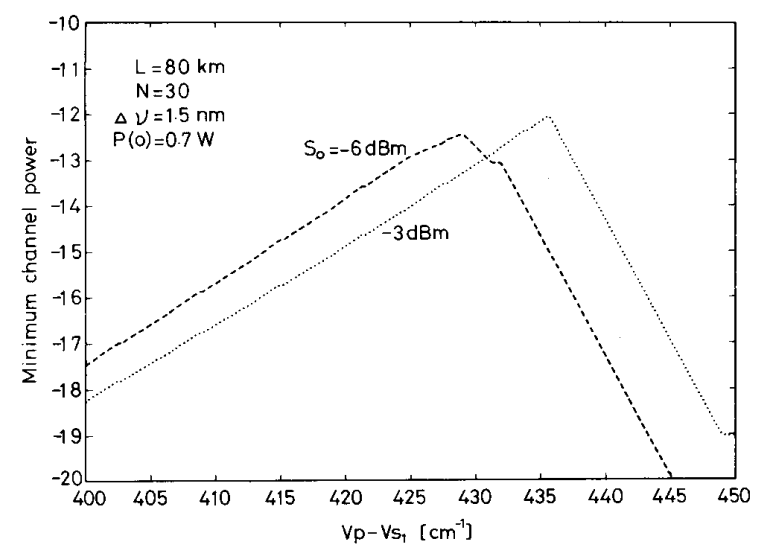

Fig. 11. The relation between the minimum received signal power and $v_{p}$ and $v_{s 1}$ for two transmitted signal levels.

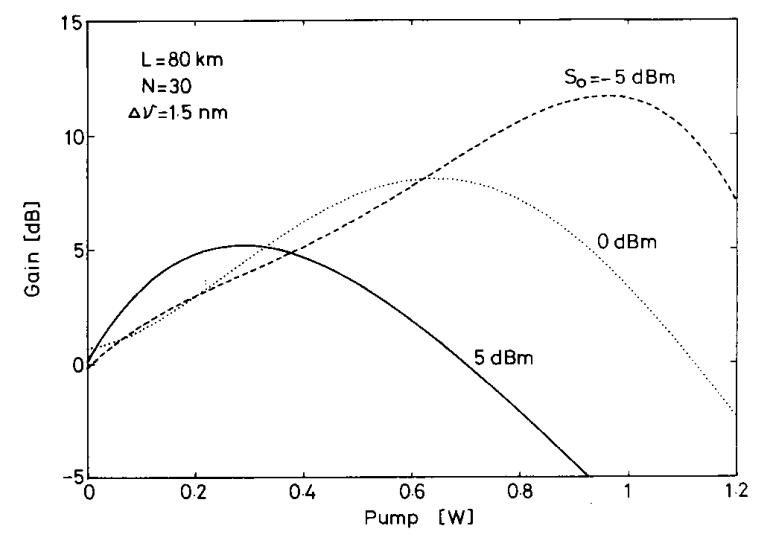

Fig. 12. Amplifier gain as a function of pump power for several $S_{0}$.

be achieved. This is due to the fact that when the pump power is larger than the optimum value, the depleted power of the least power channel may exceed that obtained from the pump so that the received power decreases.

\section{Discussion and Conclusion}

Because the transmitted signal powers are much larger than the spontaneously emitted noise powers, we have neglected the effect of spontaneous emission noise in the analysis. This noise should be included if the Raman amplifier is employed as a repeater amplifier to boost weak signals [12]. Here we are considering the use of PTFRA at the transmitting end to extend the transmission distance limitation imposed by Raman crosstalk. To further increase system transmission distance one can employ a backward FRA at the receiving end [12], [16]. Since the signal power is small compared to that at the transmitting end, the amplifier gain is expected to be higher with a backward FRA.

For illustrative purpose we consider two ways to allocate the signals within the pump gain profile. The arrangement shown in Fig. 7(a) which with an eye on fully utilizing the pump power has proven to have little advantage. In contrast, by allocating $\mathrm{CH} 1$ at the pump gain peak the received signal power is increased when low signal power is transmitted. Therefore the 


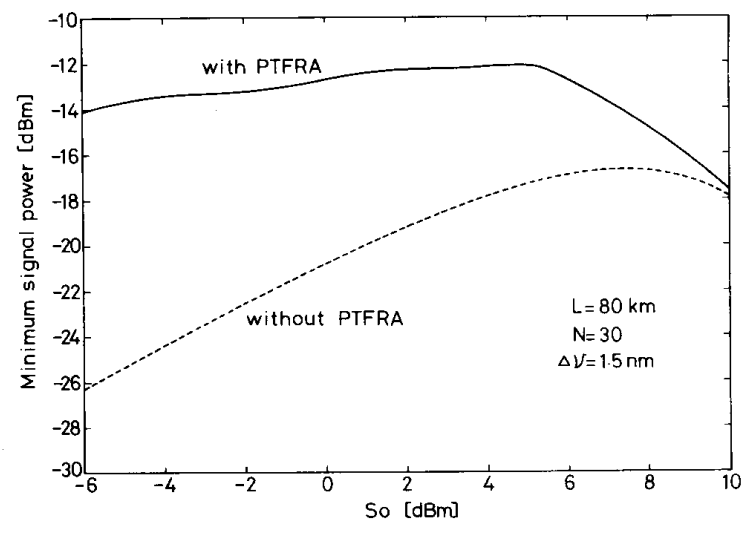

Fig. 13. The received versus transmitted signal powers for the least power channel with and without PTFRA when optimum pump power is used.

system transmission distance is extended. Such an arrangement can be achieved by properly choosing the pump frequency. As discussed before, the latter arrangement is in fact not the optimum choice, however, it gives a clear indication in choosing pumping source. For a system with given number of channels, channel spacing, as well as transmitted signal powers, numerical analysis should be carried to find the optimum channel allocation.

Fig. 13 shows the achievable signal power at $z=L$ for the least power channel with and without a PTFRA. Here, corresponding to every transmitted signal level optimum pump power is employed and the signals are allocated within the pump gain profile as Fig. 7(b). Inspection of the figure reveals that about -14- and $-26-\mathrm{dBm}$ signal powers are respectively received with and without a PTFRA when $S_{0}=-6 \mathrm{dBm}$. Thus $12-\mathrm{dB}$ gain is achieved. The maximum received power for $\mathrm{CH} 1$ without a PTFRA is about $-17 \mathrm{dBm}$ when $S_{0}=7 \mathrm{dBm}$. It is seen that with a PTFRA we can easily obtain higher signal power than the maximum value obtained without a PTFRA at a much lower transmitted signal level, thus the PTFRA can be employed to increase the system transmission distance for a system with a limited transmitted signal level. For example, with a PTFRA - 14-dBm minimum signal power is achievable with $S_{0}=-6 \mathrm{dBm}$. We further see that the maximum received power with a PTFRA is $-12 \mathrm{dBm}$ at $S_{0}=5 \mathrm{dBm}$. Therefore the limited received signal power is increased by $5 \mathrm{~dB}$ which corresponding to $20-\mathrm{km}$ transmission length for $0.25-\mathrm{dB} / \mathrm{km}$ fiber loss.

In conclusion we have analyzed the behavior of the multichannel signals in an HDWDM system with the consideration of stimulated Raman scattering. The dependence of Raman crosstalk on several system parameters is present. This crosstalk can cause serious power depletion of the shortest wavelength channel so as to limit system transmission distance. We have provided a parameter to evaluate the crosstalk. Through the introduction of a post transmitter fiber Raman amplifier, the transmission distance limitation imposed by Raman crosstalk is further extended. We have discussed the allocation of signal channels within the pump gain profile. The results indicate that the PTFRA can extend the system transmission distance particularly when the transmitted signal power is limited.

\section{REFERENCES}

[1] C. Lin, "Nonlinear optics in fibers for fiber measurements and special device functions," J. Lightwave Technol., vol. LT-4, pp. 1103-1115, 1986.

[2] R. G. Smith, "Optical power handling capability of low loss optical fiber determined by stimulated Raman and Brillouin scattering," Appl. Opt., vol. 11, pp. 2489-2494, 1972.

[3] R. H. Stolen, "Nonlinearity in fiber transmission," Proc. IEEE, vol. 68 , pp. $1232-1236,1980$.

[4] H. Kobrinski et al., "Demonstration of high. capacity in the LAMBDANET architecture: A multiwavelength optical network," Electron. Lett., vol. 23, pp. 824-826, 1987.

[5] A. R. Chraplyvy, "Limitations on lightwave communications imposed by optical fiber nonlinearities," Technique Digest of Optical Fiber Commun. Conf., 1988, p. 19.

[6] A. R. Chraplyvy and P. S. Henry, "Performance degradation due to stimulated Raman scattering in wavelength-division-multiplexed optical fiber systems," Electron. Lett., vol. 19, pp. 641$643,1983$.

[7] A. R. Chraplyvy, "Optical power limits in wavelength-divisionmultiplexed systems due to stimulated Raman scattering," Electron. Lett., vol. 20, pp. 58-59, 1984.

[8] M. Nakazawa, "Highly efficient Raman amplification in a polarization preserving optical fiber,"' Appl. Phys. Lett., vol. 46, pp. $628-630,1985$.

[9] J. Hegarty, N. A. Olsson, and L. Goldner, "CW pumped Raman preamplifier in a $45 \mathrm{~km}$ long fiber transmission system operating at $1.5 \mu \mathrm{m}$ and 1 Gbit/s," Electron. Lett., vol. 21, pp. 290-292, 1985.

[10] N. Edagawa, K. Mochizuki, and Y. Iwamoto, "Simultaneous amplification of wavelength-division-multiplexed signals by a high-power semiconductor lasers,"' Electron. Lett., vol. 23, pp. 196-197, 1987

[11] M. L. Dakss and P. Melman, "Two-channel fiber Raman amplification for WDM systems,"' IEE Proc. J, vol. 135, pp. 96-100, 1988.

[12] M. S. Kao and J. Wu, "Signal light amplification by stimulated Raman scattering in an N-channel WDM optical fiber communication system," J. Lightwave Technol., vol. 7, pp. 1290-1299, 1989.

[13] R. H. Stolen and E. P. Ippen, "Raman gain in glass optical waveguides," Appl. Phys. Lett., vol. 22, pp. 276-278, 1973.

[14] G. P. Agrawal, Nonlinear Fiber Optics. London: Academic, 1989.

[15] R. H. Stolen, "Polarization effects in fiber Raman and Brillouin lasers," IEEE J. Quantum Electron., vol. QE-15, pp. 1157-1160, 1979.

[16] K. Mochizuki, "Optical fiber transmission systems using stimulated Raman scattering: Theory,' J. Lightwave Technol., vol. LT-3, pp. 688-694, 1985.

Ming-Seng Kao, photograph and biography not available at the time of publication

Jingshown $\mathbf{W u}$, photograph and biography not available at the time of publication 